\title{
On quantum bit coding by Gaussian beam modes for the quantum key distribution
}

\author{
M. P. Faleeva, I. Y. Popov \\ ITMO University, Kronverkskiy, 49, Saint Petersburg, 197101, Russia \\ popov1955@gmail.com
}

DOI 10.17586/2220-8054-2020-11-6-651-658

\begin{abstract}
This paper deals with possibility of implementation of quantum key distribution algorithm through turbulent atmosphere. Beam wandering is considered as the main perturbation. For description of the density matrix, the Glauber-Sudarshan P-function technique is used. The probabilities of detectors triggering are determined.
\end{abstract}

Keywords: quantum bit, gaussian beam, entanglement, quantum key distribution.

Received: 30 November 2020

\section{Introduction}

Nanoscience progress opens new opportunities in the development of quantum technologies, particularly, quantum computing and quantum communications. We are studying the quantum key distribution algorithm implementation in a turbulent atmosphere. There are a variety of optical techniques for implementing quantum key distribution [1]. The most common class of solutions encode each bit of private information onto discrete degrees of freedom of optical signals, and it is named as discrete-variable quantum key distribution. An alternative approach employs coherent communication techniques to encode the private information, and is known as continuous-variable quantum key distribution. Both approaches face a similar obstacle when attempting to implement wide-scale deployment of quantum key distribution: physical communication channels introduce transmission losses that increase exponentially with distance, greatly limiting the secure key rates that can be achieved over long ranges [2-4].

To be more specific, we are interested in the protocol, based on the entangled qubit pairs (EPR-Protocol). Qubits in our research are coded by Gaussian beam modes, which are sent to the receiver. We took some simplifications for the mathematical description of the turbulent atmosphere. We focus on the effect of beam wandering, which is dominant for the weak turbulence. Beam wandering is usually truncated by the aperture at the receivers, thus beam losses appear [5]. Passed light is evaluated by the transmission coefficient. The expression of the density operator of the quantum state after passing the receiver aperture can be obtained with the use of the input-output relations in terms of the Glauber-Sudarshan P-function. Our aim is to calculate probabilities of the detectors going off occurring the concrete detector in another quantum channel goes off.

\section{Qubit representation}

Let us introduce representation of a qubit through Gaussian beam modes. Assume that a photon can be in one of two fixed states (modes) with indices $m_{1}, n_{1}$ and $m_{2}, n_{2}, m_{1}, n_{1}, m_{2}, n_{2} \in\{\mathbb{N} \cup 0\},\left(m_{1}, n_{1}\right) \neq\left(m_{2}, n_{2}\right)$. So the qubit can be represented as a linear combination of two states. We write a photon state in the form of Fock state $|10\rangle$, where " 1 " is at the first position means that the photon is in the mode $\Psi_{m 1 n 1}$. Let this state be the first basic state of the computational qubit. Correspondingly, the photon state $|01\rangle$, which means that the photon is in the mode $\Psi_{m 2 n 2}$, be the another basic state of the computational qubit. The mode set is orthonormal. The qubit can be represented as a linear combination of two states $\alpha|10\rangle+\beta|01\rangle$. We need an entangled two-qubit state, so we take four modes for two photons $\Psi_{\text {mini }}, i=1,2,3,4$. For example, two " 1 " in the notation of two-photons state $|0110\rangle$ mean that the first photon is in the mode $\Psi_{m 2 n 2}$, the second photon is in the mode $\Psi_{m 3 n 3}$.

\section{The scheme of the algorithm}

Figure 1 presents the idea of the cryptographic installation implementation. The source of EPR-pairs (E) generates an entangled pair of photons. If we use modes $\Psi_{m 1, n 1}, \Psi_{m 2, n 2}$ for qubit A coding and modes $\Psi_{m 3, n 3}, \Psi_{m 4, n 4}$ for qubit $\mathrm{B}$, the EPR-pair of entangled qubits, generated by source is given by:

$|\Phi\rangle_{i n}=\frac{1}{\sqrt{2}}\left(|1\rangle_{m 1, n 1}|0\rangle_{m 2, n 2}|0\rangle_{m 3, n 3}|1\rangle_{m 4, n 4}-|0\rangle_{m 1, n 1}|1\rangle_{m 2, n 2}|1\rangle_{m 3, n 3}|0\rangle_{m 4, n 4}\right)=\frac{1}{\sqrt{2}}(|1001\rangle-|0110\rangle)$.

One photon from the pair is sent into the channel A and another into the channel B through the atmosphere. After passing the paths $\mathrm{A}$ and $\mathrm{B}$, the corresponding photon comes into the measuring equipment, which consists in 
symmetric beam splitter (S), four filters $\left(F_{i}, i=1,2,3,4\right)$ for each channel and detectors $\left(D^{i}, i=1,2,3,4\right)$, which are assumed to be perfect. There are four nonorthogonal states, which are used in quantum key distribution protocol. We noted them in a different way for the qubit $\mathrm{A}$ and qubit B:

$$
\begin{gathered}
|\Psi\rangle_{1}^{A}=|1\rangle_{m 1, n 1}|0\rangle_{m 2, n 2}=|10\rangle_{A}, \\
|\Psi\rangle_{2}^{A}=|0\rangle_{m 1, n 1}|1\rangle_{m 2, n 2}=|01\rangle_{A}, \\
|\Psi\rangle_{3}^{A}=\frac{1}{\sqrt{2}}\left(|10\rangle_{A}+|01\rangle_{A}\right), \\
|\Psi\rangle_{4}^{A}=\frac{1}{\sqrt{2}}\left(|01\rangle_{A}-|10\rangle_{A}\right), \\
|\Psi\rangle_{1}^{B}=|1\rangle_{m 3, n 3}|0\rangle_{m 4, n 4}=|10\rangle_{B}, \\
|\Psi\rangle_{2}^{B}=|0\rangle_{m 3, n 3}|1\rangle_{m 4, n 4}=|01\rangle_{B}, \\
|\Psi\rangle_{3}^{B}=\frac{1}{\sqrt{2}}\left(|10\rangle_{B}+|01\rangle_{B}\right), \\
|\Psi\rangle_{4}^{B}=\frac{1}{\sqrt{2}}\left(|01\rangle_{B}-|10\rangle_{B}\right) .
\end{gathered}
$$

Filters distinguish pairs of orthogonal states, so the $F_{1}^{A / B}$ let the state $|\Psi\rangle_{1}^{A / B}$ pass, and reflects the state $|\Psi\rangle_{2}^{A / B}$, $F_{2}^{A / B}$ let the state $|\Psi\rangle_{3}^{A / B}$ pass, and reflects the state $|\Psi\rangle_{4}^{A / B}$.

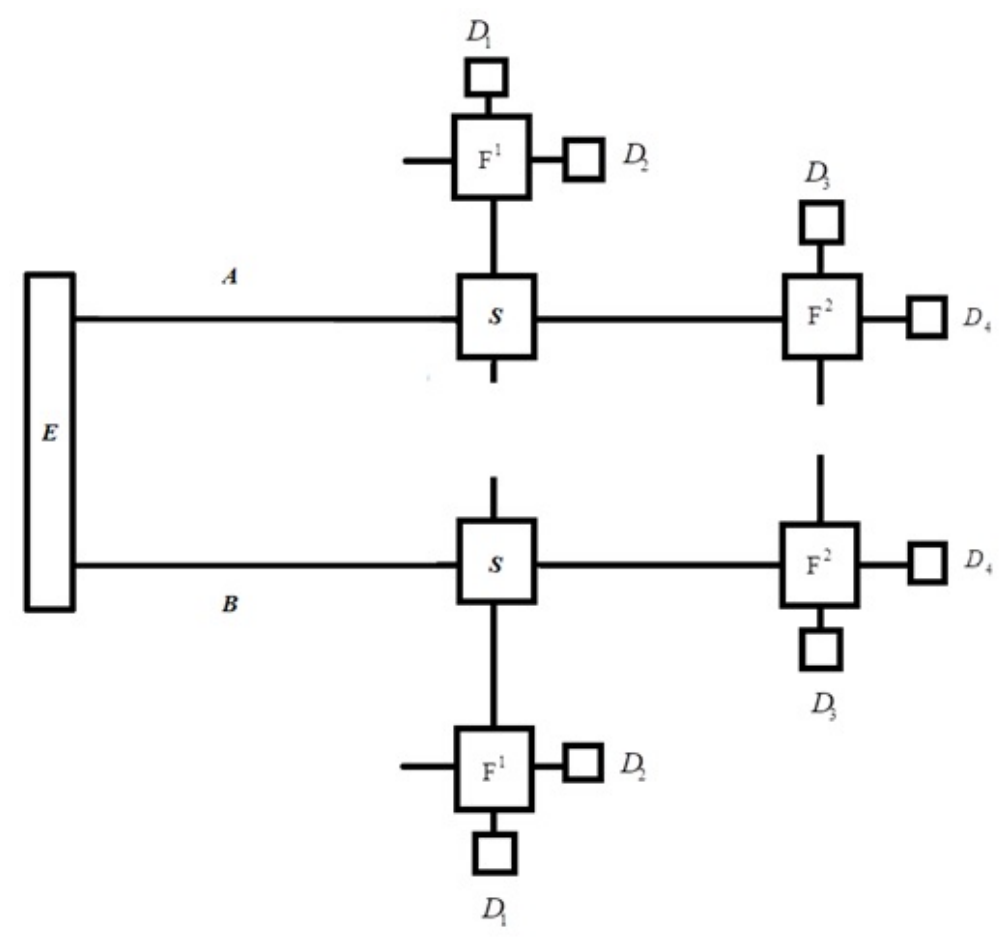

FIG. 1. The idea of the cryptographic installation scheme 


\section{Stochastic Helmholtz equation}

Generally speaking, the atmospheric turbulence can be expressed in random nature of the refraction index in a classical Helmholtz equation. It has been shown [6] that the Maxwell's equations for the amplitude of a propagating electromagnetic wave lead to the scalar stochastic Helmholtz equation for a component of field, where dependence on time, in the form of the monochromatic wave, is taken into account:

$$
\Delta U(x, y, z)+k^{2} n^{2}(x, y, z) U(x, y, z)=0
$$

where $k$ is the wave number of the electromagnetic wave, $n(x, y, z)$ is the index of refraction, which is varies due to random properties of media and it can be expressed as $n(x, y, z)=n_{0}+n_{1}(x, y, z), n_{0}=1$ is the mean index of refraction, $n_{1}(x, y, z)$ is the random deviation of index from its mean value, and it is delta correlated in the direction of the propagation.

We fix the exponential variation of $U$ in respect to the coordinate $z$, which corresponds to the direction of the beams propagation:

$$
U(x, y, z ; k)=\Psi(x, y, z ; k) \exp (i k z) .
$$

Substitution of function (11) into the equation (10) with taking the paraxial approximation leads to the paraxial form of the Helmholtz equation for the function $\Psi$ :

$$
\left(i \frac{\partial}{\partial z}+\frac{1}{2 k} \Delta_{\perp}+k n_{1}(x, y, z)\right) \Psi_{\text {in } / \text { out }}(x, y, z ; k)=0
$$

where $\Delta_{\perp}$ is the transverse part of the Laplacian: $\Delta_{\perp}=\frac{\partial^{2}}{\partial x^{2}}+\frac{\partial^{2}}{\partial y^{2}}$.

We find the eigenfunction of (12) in the same way as in [7], but we are interested in modes of high order:

$$
\begin{aligned}
& \Psi_{m n}\left(x, y, z_{a p}, k\right)= \\
& =\sqrt{\frac{2}{\pi W^{2}}} \exp \left(-\left((x-r)^{2}+y^{2}\right)\left(\frac{1}{W^{2}}+\frac{i k}{2 R}\right)+i(1+m+n) \phi\right) H_{m}\left(\frac{\sqrt{2}(x-r)}{W}\right) H_{n}\left(\frac{\sqrt{2} y}{W}\right),
\end{aligned}
$$

where $W\left(z_{a p}\right)$ is the beam-spot radius, $R\left(z_{a p}\right)$ is the curvature radius of the wavefront, $\phi\left(z_{a p}\right)$ is the Gouy phase, $H_{m}(\xi)$ is the Hermite function, where $z_{a p}$ is the position of the aperture plane at the Z-axis.

Correspondingly, for $z_{a p}=0$ we obtain the condition:

$$
\Psi_{m n}(x, y, 0, k)=\sqrt{\frac{2}{\pi W_{0}^{2}}} \exp \left(-\left(x^{2}+y^{2}\right)\left(\frac{1}{W_{0}^{2}}+\frac{i k}{2 R_{0}}\right)+i(1+m+n) \phi_{0}\right) H_{m}\left(\frac{\sqrt{2}(x)}{W_{0}}\right) H_{n}\left(\frac{\sqrt{2} y}{W_{0}}\right)
$$

where $W_{0}, R_{0}, \phi_{0}$ are initial values for the $W(z), R(z), \phi(z)$.

Parameter $r$ is the randomly generated distance of beam-deflection from the aperture center. One of the main problems for description of free space light propagation is finding the probability distribution of this parameter. Gaussian distribution approximates the index of refraction $n_{1}(x, y, z)$ quite well for the case of week turbulence [6-8]. Then, the Rice distribution is the probability distribution function for $r$ is:

$$
f(r ; d, \sigma)=\frac{r}{\sigma^{2}} I_{0}\left(\frac{r d}{\sigma^{2}}\right) \exp \left(-\frac{r^{2}+d^{2}}{2 \sigma^{2}}\right),
$$

where $I_{0}(x)$ is a modified Bessel function of the first kind and order zero, $d$ is the distance between the aperture and the fluctuation centers, $\sigma$ is the standard deviation of the beam deflection, $\sigma^{2}=1,23 C_{n}^{2} k^{7 / 6} L^{11 / 6}$, where $L$ is the path length of propagated light, $C_{n}^{2}$ is the refractive-index structure parameter. For weak fluctuations $\sigma^{2}<1$ [6].

\section{Gauss beam modes propagation}

We will analyze the propagation of light through a turbulent atmosphere for the quantum entangled two-photon state (EPR-pair) (1). The density operator of the photons state is:

$$
\widehat{\rho}_{i n}=|\Phi\rangle_{i n}\left\langle\left.\Phi\right|_{i n} .\right.
$$

To obtain the density operator for the state of the transmitted light, we will use the approach of the input-output relations in terms of the Glauber-Sudarshan P-function. This approach was applied for deriving the output density matrices of the transmitted light in turbulent atmosphere by the authors of work [9].

The well-known Glauber-Sudarshan P-function is defined by determining the form of the density operator $\hat{\rho}$ in the basis of coherent states $\{|\alpha\rangle\}$ :

$$
\hat{\rho}=\int P(\alpha)|\alpha\rangle\langle\alpha| d^{2} \alpha
$$


The next formula for the P-function is more important for the practical calculations:

$$
P(\alpha)=\frac{1}{\pi^{2}} \int_{-\infty}^{+\infty} \chi(\beta) \exp \left(\alpha \beta^{*}-\alpha^{*} \beta\right) d^{2} \beta .
$$

Here, $\chi(\beta)=\operatorname{Tr}\left[\hat{\rho} \exp \left(\hat{a}^{+} \beta\right) \exp \left(-\hat{a} \beta^{*}\right)\right]$ is the characteristic function; $\hat{a}, \hat{a}^{+}$are the annihilation and creation operators.

Relations for the above functions between the input state and the transmitted through turbulent media state are given by the following expressions:

$$
\begin{gathered}
\widehat{\rho}_{\text {out }}=\left\langle\widehat{\rho}_{T}\right\rangle, \\
\widehat{\rho}_{T}=\int P_{T}(\alpha)|\alpha\rangle\langle\alpha| d^{2} \alpha, \\
P_{T}(\alpha)=\frac{1}{T^{2}} P_{i n}\left(\frac{\alpha}{T}\right), \\
\chi_{T}(\beta)=\chi_{i n}(T \beta),
\end{gathered}
$$

where $\langle\ldots\rangle$ means averaging with a probability distribution function of the transmission coefficient $f(r ; d, \sigma)$, which comes to averaging with a probability distribution function of the beam-deflection distance $r$ :

$$
\langle g(r)\rangle=\int_{0}^{\infty} f(r ; d, \sigma) g[T(r)] d r .
$$

Formulas (16)-(21) allow one to derive the expression for the output density operator of the state (15):

$$
\begin{aligned}
\widehat{\rho}_{\text {out }}=\frac{1}{2}((\langle(1 & \left.\left.\left.-\left|T_{m 1, n 1}\right|^{2}\right)\left(1-\left|T_{m 4, n 4}\right|^{2}\right)\right\rangle+\left\langle\left(1-\left|T_{m 2, n 2}\right|^{2}\right)\left(1-\left|T_{m 3, n 3}\right|^{2}\right)\right\rangle\right) \widehat{\rho}_{0} \\
& +\left\langle\left(1-\left|T_{m 1, n 1}\right|^{2}\right)\left|T_{m 4, n 4}\right|^{2}\right\rangle \widehat{\rho}_{m 4, n 4}+\left\langle\left(1-\left|T_{m 2, n 2}\right|^{2}\right)\left|T_{m 3, n 3}\right|^{2}\right\rangle \widehat{\rho}_{m 3, n 3} \\
& +\left\langle\left(1-\left|T_{m 3, n 3}\right|^{2}\right)\left|T_{m 2, n 2}\right|^{2}\right\rangle \widehat{\rho}_{m 2, n 2}+\left\langle\left(1-\left|T_{m 4, n 4}\right|^{2}\right)\left|T_{m 1, n 1}\right|^{2}\right\rangle \widehat{\rho}_{m 1, n 1} \\
& \left.+\left(\left\langle\left|T_{m 1, n 1}\right|^{2}\left|T_{m 4, n 4}\right|^{2}\right\rangle+\left\langle\left|T_{m 2, n 2}\right|^{2}\left|T_{m 3, n 3}\right|^{2}\right\rangle\right) \widehat{\rho}_{2}\right) .
\end{aligned}
$$

Here, $T_{m i, n i}$ is the transmission coefficient of the mode with indexes $m i, n i, \widehat{\rho}_{0}$ is the density operator of vacuum state, $\widehat{\rho}_{m i, n i}, i=1,2,3,4$ are the density operator of one photon states, $\widehat{\rho}_{2}$ is the density operator of two photon states.

The expression (23) can't be written in any basis. But in the case of absence of the background radiation and dark counts we can take into account only contribution of the last term in (23). This term can be represented in a matrix form in the basis of states $|0101\rangle,|1001\rangle,|0110\rangle,|1010\rangle$ :

$$
\widehat{\rho}_{2}=\frac{1}{A+C}\left[\begin{array}{llll}
0 & 0 & 0 & 0 \\
0 & A & B & 0 \\
0 & B^{\prime} & C & 0 \\
0 & 0 & 0 & 0
\end{array}\right]
$$

where $A=\left\langle\left|T_{m 1, n 1}\right|^{2}\left|T_{m 4, n 4}\right|^{2}\right\rangle, B=-\left\langle T_{m 1, n 1}^{*} T_{m 2, n 2} T_{m 1, n 1} T_{m 4, n 4}^{*}\right\rangle, B^{\prime}=-\left\langle T_{m 1, n 1} T_{m 2, n 2}^{*} T_{m 3, n 3}^{*} T_{m 4, n 4}\right\rangle$, $C=\left\langle\left|T_{m 2, n 2}\right|^{2}\left|T_{m 3, n 3}\right|^{2}\right\rangle$.

The transmission coefficients are calculated in such a way:

$$
T_{m, n}^{2} \approx T_{m, n}^{2}\left(k_{0}\right)=\int_{A}\left|U_{m, n}\left(x, y, z_{a p} ; k_{0}\right)\right|^{2} d x d y,
$$

where $A$ is the area of aperture opening.

We need to find all components of the matrix (24). It is convenient to evaluate integral (25) in polar coordinates:

$$
\begin{aligned}
& T_{m, n}^{2}(r) \approx \int_{A}\left|U_{m, n}\left(\rho, \phi, z_{a p} ; k_{0}\right)\right|^{2} d \rho d \phi \\
= & \frac{2}{\pi W^{2}} \exp \left(-\frac{2 r^{2}}{W^{2}}\right) \int_{0}^{a} \rho \exp \left(-\frac{2 \rho^{2}}{W^{2}}\right) \int_{0}^{2 \pi} \exp \left(\frac{4 \pi \rho \cos \phi}{W^{2}}\right) H_{m}^{2}\left(\frac{\sqrt{2}(\rho \cos \phi-r)}{W}\right) H_{n}^{2}\left(\frac{\sqrt{2} \rho \sin \phi}{W}\right) d \phi d \rho .
\end{aligned}
$$




\section{The qubits measurement}

Let us describe all the ways of the density operator $\widehat{\rho}_{2}$ transformation when the qubits pass the filter and get on the detectors.

In our consideration, modes $\Psi_{m 1, n 1}$ and $\Psi_{m 2, n 2}$ define basic qubit state of the qubit A (noted above by $|10\rangle_{A}$ and $\left.|01\rangle_{A}\right)$. The states (2)-(9) can be associated with the matrix representation in a standard way:

$$
\begin{gathered}
|\Psi\rangle_{1}^{A}=|10\rangle_{A} \rightarrow\left[\begin{array}{l}
1 \\
0
\end{array}\right]_{A}|\Psi\rangle_{2}^{A}=|01\rangle_{A} \rightarrow\left[\begin{array}{l}
0 \\
1
\end{array}\right]_{A}, \\
|\Psi\rangle_{3}^{A}=\frac{1}{\sqrt{2}}\left(|10\rangle_{A}+|01\rangle_{A}\right) \rightarrow \frac{1}{\sqrt{2}}\left[\begin{array}{l}
1 \\
1
\end{array}\right]_{A}, \quad|\Psi\rangle_{4}^{A}=\frac{1}{\sqrt{2}}\left(|01\rangle_{A}-|10\rangle_{A}\right) \rightarrow \frac{1}{\sqrt{2}}\left[\begin{array}{l}
-1 \\
1
\end{array}\right]_{A} .
\end{gathered}
$$

Analogously, for modes $\Psi_{m 3, n 3}$ and $\Psi_{m 4, n 4}$, one defines the basic qubit state of the qubit B:

$$
\begin{gathered}
|\Psi\rangle_{1}^{B}=|10\rangle_{B} \rightarrow\left[\begin{array}{l}
1 \\
0
\end{array}\right]_{B}, \quad|\Psi\rangle_{2}^{B}=|01\rangle_{B} \rightarrow\left[\begin{array}{l}
0 \\
1
\end{array}\right]_{B}, \\
|\Psi\rangle_{3}^{B}=\frac{1}{\sqrt{2}}\left(|10\rangle_{B}+|01\rangle_{B}\right) \rightarrow \frac{1}{\sqrt{2}}\left[\begin{array}{l}
1 \\
1
\end{array}\right]_{B}, \quad|\Psi\rangle_{4}^{B}=\frac{1}{\sqrt{2}}\left(|01\rangle_{B}-|10\rangle_{B}\right) \rightarrow \frac{1}{\sqrt{2}}\left[\begin{array}{l}
-1 \\
1
\end{array}\right]_{B} .
\end{gathered}
$$

The density operator of two-qubit state after the qubit A passing through the filter $F_{i}^{A}$, can be obtained by the projection operator $\widehat{D}_{i}^{A}$ on the state $|\Psi\rangle_{i}^{A}$ applying [9]:

$$
\begin{aligned}
& \widehat{D}_{i}=|\Psi\rangle_{i}^{A}\left\langle\left.\Psi\right|_{i} ^{A} \otimes I,\right. \\
& \widehat{D}_{1}^{A}=\left[\begin{array}{l}
1 \\
0
\end{array}\right]_{A} \cdot\left[\begin{array}{ll}
1 & 0
\end{array}\right]_{A} \otimes\left[\begin{array}{ll}
1 & 0 \\
0 & 1
\end{array}\right]=\left[\begin{array}{ll}
1 & 0 \\
0 & 0
\end{array}\right] \otimes\left[\begin{array}{ll}
1 & 0 \\
0 & 1
\end{array}\right]=\left[\begin{array}{llll}
1 & 0 & 0 & 0 \\
0 & 1 & 0 & 0 \\
0 & 0 & 0 & 0 \\
0 & 0 & 0 & 0
\end{array}\right], \\
& \widehat{D}_{2}^{A}=\left[\begin{array}{l}
0 \\
1
\end{array}\right]_{A} \cdot\left[\begin{array}{ll}
0 & 1
\end{array}\right]_{A} \otimes\left[\begin{array}{ll}
1 & 0 \\
0 & 1
\end{array}\right]=\left[\begin{array}{llll}
0 & 0 & 0 & 0 \\
0 & 0 & 0 & 0 \\
0 & 0 & 1 & 0 \\
0 & 0 & 0 & 1
\end{array}\right] \\
& \widehat{D}_{3}^{A}=\frac{1}{2} \cdot\left[\begin{array}{l}
1 \\
1
\end{array}\right]_{A} \cdot\left[\begin{array}{ll}
1 & 1
\end{array}\right]_{A} \otimes\left[\begin{array}{ll}
1 & 0 \\
0 & 1
\end{array}\right]=\frac{1}{2} \cdot\left[\begin{array}{llll}
1 & 0 & 1 & 0 \\
0 & 1 & 0 & 1 \\
1 & 0 & 1 & 0 \\
0 & 1 & 0 & 1
\end{array}\right] \\
& \widehat{D}_{4}^{A}=\frac{1}{2} \cdot\left[\begin{array}{l}
-1 \\
1
\end{array}\right]_{A} \cdot\left[\begin{array}{ll}
-1 & 1
\end{array}\right]_{A} \otimes\left[\begin{array}{ll}
1 & 0 \\
0 & 1
\end{array}\right]=\frac{1}{2} \cdot\left[\begin{array}{llll}
1 & 0 & -1 & 0 \\
0 & 1 & 0 & -1 \\
-1 & 0 & 1 & 0 \\
0 & -1 & 0 & 1
\end{array}\right] .
\end{aligned}
$$

Let us apply the operators (26)-(29) to the density operator $\widehat{\rho}_{2}$ :

$$
\begin{aligned}
& \widehat{D}_{1}^{A} \widehat{\rho}_{2}\left(\widehat{D}_{1}^{A}\right)^{*}=\frac{1}{A+C}\left[\begin{array}{cccc}
1 & 0 & 0 & 0 \\
0 & 1 & 0 & 0 \\
0 & 0 & 0 & 0 \\
0 & 0 & 0 & 0
\end{array}\right] \cdot\left[\begin{array}{llll}
0 & 0 & 0 & 0 \\
0 & A & B & 0 \\
0 & B^{\prime} & C & 0 \\
0 & 0 & 0 & 0
\end{array}\right] \cdot\left[\begin{array}{llll}
1 & 0 & 0 & 0 \\
0 & 1 & 0 & 0 \\
0 & 0 & 0 & 0 \\
0 & 0 & 0 & 0
\end{array}\right]=\frac{1}{A+C}\left[\begin{array}{cccc}
0 & 0 & 0 & 0 \\
0 & A & 0 & 0 \\
0 & 0 & 0 & 0 \\
0 & 0 & 0 & 0
\end{array}\right], \\
& \widehat{D}_{2}^{A} \widehat{\rho}_{2}\left(\widehat{D}_{2}^{A}\right)^{*}=\frac{1}{A+C}\left[\begin{array}{llll}
0 & 0 & 0 & 0 \\
0 & 0 & 0 & 0 \\
0 & 0 & 1 & 0 \\
0 & 0 & 0 & 1
\end{array}\right] \cdot\left[\begin{array}{llll}
0 & 0 & 0 & 0 \\
0 & A & B & 0 \\
0 & B^{\prime} & C & 0 \\
0 & 0 & 0 & 0
\end{array}\right] \cdot\left[\begin{array}{llll}
0 & 0 & 0 & 0 \\
0 & 0 & 0 & 0 \\
0 & 0 & 1 & 0 \\
0 & 0 & 0 & 1
\end{array}\right]=\frac{1}{A+C}\left[\begin{array}{llll}
0 & 0 & 0 & 0 \\
0 & 0 & 0 & 0 \\
0 & 0 & B^{\prime} & 0 \\
0 & 0 & 0 & 0
\end{array}\right],
\end{aligned}
$$




$$
\begin{aligned}
& \widehat{D}_{3}^{A} \widehat{\rho}_{2}\left(\widehat{D}_{3}^{A}\right)^{*}=\frac{1}{4(A+C)}\left[\begin{array}{llll}
1 & 0 & 1 & 0 \\
0 & 1 & 0 & 1 \\
1 & 0 & 1 & 0 \\
0 & 1 & 0 & 1
\end{array}\right] \cdot\left[\begin{array}{llll}
0 & 0 & 0 & 0 \\
0 & A & B & 0 \\
0 & B^{\prime} & C & 0 \\
0 & 0 & 0 & 0
\end{array}\right] \cdot\left[\begin{array}{llll}
1 & 0 & 1 & 0 \\
0 & 1 & 0 & 1 \\
1 & 0 & 1 & 0 \\
0 & 1 & 0 & 1
\end{array}\right] \\
& =\frac{1}{4(A+C)}\left[\begin{array}{llll}
C & B^{\prime} & C & B^{\prime} \\
B & A & B & A \\
C & B^{\prime} & C & B^{\prime} \\
B & A & B & A
\end{array}\right], \\
& \widehat{D}_{4}^{A} \widehat{\rho}_{2}\left(\widehat{D}_{4}^{A}\right)=\frac{1}{4(A+C)}\left[\begin{array}{llll}
1 & 0 & -1 & 0 \\
0 & 1 & 0 & -1 \\
-1 & 0 & 1 & 0 \\
0 & -1 & 0 & 1
\end{array}\right] \cdot\left[\begin{array}{llll}
0 & 0 & 0 & 0 \\
0 & A & B & 0 \\
0 & B^{\prime} & C & 0 \\
0 & 0 & 0 & 0
\end{array}\right] \cdot\left[\begin{array}{llll}
1 & 0 & -1 & 0 \\
0 & 1 & 0 & -1 \\
-1 & 0 & 1 & 0 \\
0 & -1 & 0 & 1
\end{array}\right] \\
& =\frac{1}{4(A+C)}\left[\begin{array}{llll}
C & -B^{\prime} & -C & B^{\prime} \\
-B & A & B & -A \\
-C & B^{\prime} & C & -B^{\prime} \\
B & -A & -B & A
\end{array}\right] \text {. }
\end{aligned}
$$

Then we normed the obtained matrixes in such a way:

$$
\widehat{\rho}_{2}^{(i)}=\frac{1}{\operatorname{Tr}\left(\widehat{D}_{i}^{A} \widehat{\rho}_{B}\left(\widehat{D}_{i}^{A}\right)\right)}\left(\widehat{D}_{i}^{A} \widehat{\rho}_{2}\left(\widehat{D}_{i}^{A}\right)\right) .
$$

Now, we come to the following density operators:

$$
\begin{gathered}
\widehat{\rho}_{2}^{(1)}=\frac{1}{A}\left[\begin{array}{llll}
0 & 0 & 0 & 0 \\
0 & A & 0 & 0 \\
0 & 0 & 0 & 0 \\
0 & 0 & 0 & 0
\end{array}\right], \quad \widehat{\rho}_{2}^{(2)}=\frac{1}{B^{\prime}}\left[\begin{array}{llll}
0 & 0 & 0 & 0 \\
0 & 0 & 0 & 0 \\
0 & 0 & B^{\prime} & 0 \\
0 & 0 & 0 & 0
\end{array}\right], \\
\widehat{\rho}_{2}^{(3)}=\frac{1}{2(A+C)}\left[\begin{array}{llll}
C & B^{\prime} & C & B^{\prime} \\
B & A & B & A \\
C & B^{\prime} & C & B^{\prime} \\
B & A & B & A
\end{array}\right], \quad \widehat{\rho}_{2}^{(4)}=\frac{1}{2(A+C)}\left[\begin{array}{llll}
C & -B^{\prime} & -C & B^{\prime} \\
-B & A & B & -A \\
-C & B^{\prime} & C & -B^{\prime} \\
B & -A & -B & A
\end{array}\right] .
\end{gathered}
$$

The reduced density matrix of the qubit $\mathrm{B}$ after the qubit $\mathrm{A}$ is measurement can be calculated by taking the partial trace over system A:

$$
\begin{gathered}
\widehat{\rho}_{B}^{(i)}=\operatorname{Tr}_{A}\left(\widehat{\rho}_{2}^{(i)}\right) \\
=\left(\left[\begin{array}{ll}
1 & 0
\end{array}\right]_{A} \otimes\left[\begin{array}{ll}
1 & 0 \\
0 & 1
\end{array}\right]\right) \widehat{\rho}_{2}^{(i)}\left(\left[\begin{array}{l}
1 \\
0
\end{array}\right]_{A} \otimes\left[\begin{array}{ll}
1 & 0 \\
0 & 1
\end{array}\right]\right)+\left(\left[\begin{array}{ll}
0 & 1
\end{array}\right]_{A} \otimes\left[\begin{array}{ll}
1 & 0 \\
0 & 1
\end{array}\right]\right) \widehat{\rho}_{2}^{(i)}\left(\left[\begin{array}{l}
0 \\
1
\end{array}\right]_{A} \otimes\left[\begin{array}{ll}
1 & 0 \\
0 & 1
\end{array}\right]\right) \\
=\left[\begin{array}{llll}
1 & 0 & 0 & 0 \\
0 & 1 & 0 & 0
\end{array}\right] \widehat{\rho}_{2}^{(i)}\left[\begin{array}{ll}
1 & 0 \\
0 & 1 \\
0 & 0 \\
0 & 0
\end{array}\right]+\left[\begin{array}{llll}
0 & 0 & 1 & 0 \\
0 & 0 & 0 & 1
\end{array}\right] \widehat{\rho}_{2}^{(i)}\left[\begin{array}{ll}
0 & 0 \\
0 & 0 \\
1 & 0 \\
0 & 1
\end{array}\right] .
\end{gathered}
$$

We get the following matrixes:

$$
\widehat{\rho}_{B}^{(1)}=\frac{1}{A}\left[\begin{array}{ll}
0 & 0 \\
0 & A
\end{array}\right], \quad \widehat{\rho}_{B}^{(2)}=\frac{1}{B^{\prime}}\left[\begin{array}{ll}
B^{\prime} & 0 \\
0 & 0
\end{array}\right]
$$




$$
\widehat{\rho}_{B}^{(3)}=\frac{1}{(A+C)}\left[\begin{array}{ll}
C & B^{\prime} \\
B & A
\end{array}\right], \quad \widehat{\rho}_{B}^{(4)}=\frac{1}{(A+C)}\left[\begin{array}{ll}
C & -B^{\prime} \\
-B & A
\end{array}\right] .
$$

Now, we can calculate the probabilities of the detectors $D_{B}^{j}$ triggering occurring given that detectors $D_{A}^{i}$ is triggering:

$$
\begin{aligned}
& P^{i j}=\frac{1}{2}|\Psi\rangle_{j}^{B} \widehat{\rho}_{B}^{(i)}\left\langle\left.\Psi\right|_{j} ^{B},\right. \\
& P^{11}=\frac{1}{2 A}\left[\begin{array}{ll}
1 & 0
\end{array}\right] \cdot\left[\begin{array}{ll}
0 & 0 \\
0 & A
\end{array}\right]\left[\begin{array}{l}
1 \\
0
\end{array}\right]=0 \\
& P^{12}=\frac{1}{2 A}\left[\begin{array}{ll}
0 & 1
\end{array}\right] \cdot\left[\begin{array}{ll}
0 & 0 \\
0 & A
\end{array}\right]\left[\begin{array}{l}
0 \\
1
\end{array}\right]=\frac{1}{2}, \\
& P^{13}=\frac{1}{4 A}\left[\begin{array}{ll}
1 & 1
\end{array}\right] \cdot\left[\begin{array}{ll}
0 & 0 \\
0 & A
\end{array}\right]\left[\begin{array}{l}
1 \\
1
\end{array}\right]=\frac{1}{4}, \\
& P^{14}=\frac{1}{4 A}\left[\begin{array}{ll}
-1 & 1
\end{array}\right] \cdot\left[\begin{array}{ll}
0 & 0 \\
0 & A
\end{array}\right]\left[\begin{array}{l}
-1 \\
1
\end{array}\right]=\frac{1}{4}, \\
& P^{21}=\frac{1}{2 B^{\prime}}\left[\begin{array}{ll}
1 & 0
\end{array}\right] \cdot\left[\begin{array}{ll}
B^{\prime} & 0 \\
0 & 0
\end{array}\right]\left[\begin{array}{l}
1 \\
0
\end{array}\right]=\frac{1}{2}, \\
& P^{22}=\frac{1}{2 B^{\prime}}\left[\begin{array}{ll}
0 & 1
\end{array}\right] \cdot\left[\begin{array}{ll}
B^{\prime} & 0 \\
0 & 0
\end{array}\right]\left[\begin{array}{l}
0 \\
1
\end{array}\right]=0, \\
& P^{23}=\frac{1}{4 B^{\prime}}\left[\begin{array}{ll}
1 & 1
\end{array}\right] \cdot\left[\begin{array}{ll}
B^{\prime} & 0 \\
0 & 0
\end{array}\right]\left[\begin{array}{l}
1 \\
1
\end{array}\right]=\frac{1}{4}, \\
& P^{24}=\frac{1}{4 B^{\prime}}\left[\begin{array}{ll}
-1 & 1
\end{array}\right] \cdot\left[\begin{array}{ll}
B^{\prime} & 0 \\
0 & 0
\end{array}\right]\left[\begin{array}{l}
-1 \\
1
\end{array}\right]=\frac{1}{4} \\
& P^{31}=\frac{1}{2(A+C)}\left[\begin{array}{ll}
1 & 0
\end{array}\right] \cdot\left[\begin{array}{cc}
C & B^{\prime} \\
B & A
\end{array}\right]\left[\begin{array}{l}
1 \\
0
\end{array}\right]=\frac{C}{2(A+C)}, \\
& P^{32}=\frac{1}{2(A+C)}\left[\begin{array}{ll}
0 & 1
\end{array}\right] \cdot\left[\begin{array}{cc}
C & B^{\prime} \\
B & A
\end{array}\right]\left[\begin{array}{l}
0 \\
1
\end{array}\right]=\frac{A}{2(A+C)}, \\
& P^{33}=\frac{1}{4(A+C)}\left[\begin{array}{ll}
1 & 1
\end{array}\right] \cdot\left[\begin{array}{cc}
C & B^{\prime} \\
B & A
\end{array}\right]\left[\begin{array}{l}
1 \\
1
\end{array}\right]=\frac{A+B+B^{\prime}+C}{4(A+C)} \\
& P^{34}=\frac{1}{4(A+C)}\left[\begin{array}{ll}
-1 & 1
\end{array}\right] \cdot\left[\begin{array}{ll}
C & B^{\prime} \\
B & A
\end{array}\right]\left[\begin{array}{l}
-1 \\
1
\end{array}\right]=\frac{A-B-B^{\prime}+C}{4(A+C)}, \\
& P^{41}=\frac{1}{2(A+C)}\left[\begin{array}{ll}
1 & 0
\end{array}\right] \cdot\left[\begin{array}{ll}
C & -B^{\prime} \\
-B & A
\end{array}\right]\left[\begin{array}{l}
1 \\
0
\end{array}\right]=\frac{C}{2(A+C)}, \\
& P^{42}=\frac{1}{2(A+C)}\left[\begin{array}{ll}
0 & 1
\end{array}\right] \cdot\left[\begin{array}{ll}
C & -B^{\prime} \\
-B & A
\end{array}\right]\left[\begin{array}{l}
0 \\
1
\end{array}\right]=\frac{A}{2(A+C)}, \\
& P^{44}=\frac{1}{4(A+C)}\left[\begin{array}{ll}
-1 & 1
\end{array}\right] \cdot\left[\begin{array}{ll}
C & -B^{\prime} \\
-B & A
\end{array}\right]\left[\begin{array}{l}
-1 \\
1
\end{array}\right]=\frac{A+B+B^{\prime}+C}{4(A+C)}, \\
& P^{43}=\frac{1}{4(A+C)}\left[\begin{array}{ll}
1 & 1
\end{array}\right] \cdot\left[\begin{array}{ll}
C & -B^{\prime} \\
-B & A
\end{array}\right]\left[\begin{array}{l}
1 \\
1
\end{array}\right]=\frac{A-B-B^{\prime}+C}{4(A+C)} .
\end{aligned}
$$

\section{Acknowledgments}

The reported study was funded by RFBR, project number 19-31-90154. 


\section{References}

[1] Derkach I., Usenko V.C., Filip R. Squeezing-enhanced quantum key distribution over atmospheric channels. New J. Phys., 2020,22 , P. 053006.

[2] Dixon A., et al. High speed prototype quantum key distribution system and long term field trial. Opt. Express, 2015 , 23, P. 7583-7592.

[3] Choi I., et al. Field trial of a quantum secured $10 \mathrm{gb} / \mathrm{s}$ dwdm transmission system over a single installed fiber. Opt. Express, 2014, 22, P. 23121-23128.

[4] Fröhlich B., et al. Quantum secured gigabit optical access networks. Sci. Rep., 2015, 5, P. 18121.

[5] Bohmann M., Semenov A.A., Sperling J., Vogel W. Gaussian entanglement in the turbulent atmosphere. Phys. Rev. A, 2016, 94, P. 010302(R).

[6] Andrews L.C., Phillips R.L. Laser beam propagation through random media, SPIE Press, Bellingham, Washingtin, 2005.

[7] Vasylyev D.Yu., Semenov A.A., Vogel W. Toward Global Quantum Communication: Beam Wandering Preserves Nonclassicality. Phys. Rev. Lett., 2012, 108, P. 220501.

[8] Vasylyev D.Yu., Semenov A.A., Vogel W. Atmospheric Quantum Channels with Weak and Strong Turbulence. Phys. Rev. Lett., 2016, 117, P. 090501.

[9] Semenov A.A., Vogel W.Entanglement transfer through the turbulent atmosphere, Phys. Rev. A., 2010, 81, P. 023835.

[10] Miroshnichenko G.P., Sotnikova A.A. Optimization of optical fiber parameters to reduce errors of quantum key distribution using entangled polarization states of biphotons. Optics and Spectroscopy, 2012, 112, P. 327-334.

[11] Gaidash A.A.,Medmedeva S.S.,Miroshnichenko G.P. Analysis of the unambiguous state discrimination with unequal a priori probabilities. Nanosystems: Physics, Chemistry, Mathematics, 2019, 10(4), P. 398-401.

[12] Kiselev F.D., Samsonov E.Y., Gleim A.V. Modeling of linear optical controlled-Z quantum gate with dimensional errors of passive components. Nanosystems: Physics, Chemistry, Mathematics, 2019, 10(6), P. 627-631. 